\title{
ON THE STRUCTURE OF PRIMARY ABELIAN GROUPS OF COUNTABLE ULM TYPE
}

BY

\author{
DOYLE O. CUTLER
}

\begin{abstract}
In this paper we will give structure theorems for abelian p-groups of countable Ulm type utilizing the notion of high subgroup introduced by John $\mathbf{M}$. Irwin and its generalization, $N$-high subgroup, introduced by Irwin and E. A. Walker. The general technique employed is to give conditions under which automorphisms of these subgroups extend to automorphisms of the group.
\end{abstract}

In this paper we will give structure theorems for abelian $p$-groups of countable Ulm type utilizing the notion of high subgroup introduced by John M. Irwin and its generalization, $N$-high subgroup, introduced by Irwin and E. A. Walker. The general technique employed is to give conditions under which automorphisms of these subgroups extend to automorphisms of the group.

Let $G$ be a reduced abelian $p$-group and $\beta$ a limit ordinal. A chain of subgroups $\left\{G_{n}\right\}_{n \in \omega}$ of $G$ is said to be a $\beta$-high chain in $G$ if $G_{n}$ is a $p^{\beta} G$-high subgroup of $G$. If $\sigma$ is the natural homomorphism from $G$ onto $G / p^{\beta} G$, then $\left\{\sigma\left(G_{n}\right)\right\}_{n \in \omega}$ is said to be a $\beta$-high chain in $G / p^{\beta} G$. We show that if $G$ and $H$ are reduced abelian $p$-groups such that $p^{\beta} G \cong p^{\beta} H, p^{\beta} G$ is torsion complete and there exists an isomorphism between $G / p^{\beta} G$ and $H / p^{\beta} H$ preserving the $\beta$-high chains, then $G$ and $H$ are isomorphic. In the case that $p^{\beta} G$ is not torsion complete, heavy restrictions must be imposed on these isomorphisms. In particular we have that a $p$-group $G$ for which $G / p^{\omega} G$ is torsion complete and $p^{\omega} G$ is homogeneous with order bound $p^{n}$ is completely determined by $G / p^{\omega} G, p^{\omega} G$ and its class of high subgroups.

To consider groups of Ulm type $\leqq \omega$, we introduce the idea of an $\omega$-summable group: a group $C$ is said to be $\omega$-summable if $C=\bigcup_{n \in \omega} C_{n}$ such that $\left\{C_{n}\right\}_{n \in \omega}$ is a chain of $p^{\omega n} C$-high subgroups of $C$. We give necessary and sufficient conditions for two $\omega$-summable groups to be isomorphic, and show that any abelian $p$-group of Ulm type $\leqq \omega$ is an isotype subgroup between an $\omega$-summable group $C$ and its completion with respect to the topology induced by $\left\{p^{\omega n} C\right\}_{n \in \omega}$.

For groups of countable Ulm type, we get similar results using "generalized" Ulm factors (for which the groups of Ulm type $\omega$ are a special case). From this we not only show the existence of reduced abelian $p$-groups of countable Ulm type

Received by the editors July $15,1969$.

AMS 1970 subject classification. Primary 20K10.

Key words and phrases. Structure of primary abelian groups, countable Ulm type, high subgroup, $N$-high subgroup. 
having prescribed Ulm factors but obtain all such groups. This generalizes results of Fuchs and Kulikov as prescribed in Theorems 35.5 and 38.2 of [4].

Examples are then given to show that some of the structure theorems are the best possible, and to give greater insight as to why two reduced abelian $p$-groups having the same Ulm factors might differ.

In what follows, all groups will be $p$-primary abelian groups. If $\alpha$ is an ordinal, by $\beta \in \alpha$ we mean $0 \leqq \beta<\alpha, \omega$ is the first infinite ordinal, $\omega^{*}=\omega-\{0\}$, and $c$ is the cardinality of the continuum. If $G$ is a group and $a \in G$, then $\tau(G)$ will be the Ulm type of $G, r(G)$ the rank of $G$, and $h(a)$ the generalized height of $a$ in $G$ (see [4]). Also $\oplus$ and $\Sigma$ will be used for direct sum and $\langle\cdots\rangle$ for group generated by $\cdots$. In general, the notation and terminology will be similar to that of [4] or [10].

1. Preliminaries. For notational purposes we will say that a subgroup $H$ of $G$ is $\beta$-high in $G$ ( $\beta$ an ordinal) if $H$ is a $p^{\beta} G$-high subgroup of $G$ (see [7]). Thus an $\omega$-high subgroup of $G$ is just a high subgroup of $G$.

The following lemma will be very useful in the sequel.

LEMMA 1.1. Let $G$ be a reduced p-group and let $H$ be $a(\alpha+n)$-high subgroup of $G$ with $\alpha$ a limit ordinal and $n$ a nonnegative integer. Then $G\left[p^{s}\right]=H\left[p^{s}\right] \oplus K\left[p^{s}\right]$ for $s \leqq n+1$ and any complementary summand $K$ of a maximal $p^{n}$-bounded summand of $p^{\alpha} G$.

Proof. Clearly $G[p]=H[p] \oplus K[p]$. Suppose $G\left[p^{s}\right]=H\left[p^{s}\right] \oplus K\left[p^{s}\right]$ for $s<n+1$. Let $x \in G\left[p^{s+1}\right]-G\left[p^{s}\right]$. Then $p x=h+y$ such that $h \in H\left[p^{s}\right]$ and $y \in K\left[p^{s}\right]$. Since $K$ is a complementary summand of a maximal $p^{n}$-bounded summand of $p^{\alpha} G$ we may choose $z \in K$ such that $p z=y$. Since $H$ is pure we may choose $k \in H$ such that $p k=h$. Thus $x-(k+z) \in G[p]$ and the result follows.

The next theorem arose while considering the following question: Let $G$ and $H$ be groups with high subgroups $K$ and $L$, respectively. Let $\alpha: G \rightarrow G / p^{\omega} G$ and $\beta: H \rightarrow H / p^{\omega} H$ be natural. Suppose that $p^{\omega} G \cong p^{\omega} H$ and that $\gamma$ is an isomorphism from $K$ onto $L$ such that the isomorphism induced by $\gamma$ from $\alpha(K)$ onto $\beta(L)$ can be extended to an isomorphism from $G / p^{\omega} G$ onto $H / p^{\omega} H$. Are $G$ and $H$ isomorphic? If $p^{\omega} G$ is a direct sum of cyclic groups each of order $p^{n}$ then the answer is in the affirmative as indicated by the following theorem. In general the answer is negative as is shown by Example 1 in $\$ 4$.

THEOREM 1.2. Let $G$ and $H$ be reduced $p$-groups, $\delta$ a limit ordinal, and $n$ a positive integer such that

(i) there exist $(\delta+n-1)$-high subgroups $G_{n-1}$ and $H_{n-1}$ of $G$ and $H$, respectively, that are isomorphic under an isomorphism $\alpha$ and

(ii) there exist $(\delta+n)$-high subgroups $G_{n}$ and $H_{n}$ of $G$ and $H$, respectively, containing $G_{n-1}$ and $H_{n-1}$, respectively, and an isomorphism $\sigma$ from $G_{n} / p^{\delta} G_{n}$ onto $H_{n} / p^{\delta} H_{n}$ such that $\sigma\left(x+p^{\delta} G_{n}\right)=\alpha(x)+p^{\delta} H_{n}$ for all $x \in G_{n-1}$.

Then there exists an isomorphism $\beta$ from $G_{n}$ onto $H_{n}$ such that $\sigma\left(x+p^{\delta} G_{n}\right)=\beta(x)$ $+p^{\delta} H_{n}$ for all $x \in G_{n}$ and $\beta \mid G_{n-1}=\alpha$. 
Proof. Note that $p^{\delta} G_{k}=\sum_{i=1}^{k} B_{i}$ with $B_{i}=\sum_{\mu \in \Gamma_{i}}\left\langle u_{i}^{\mu}\right\rangle$ such that $O\left(u_{i}^{\mu}\right)=p^{i}$ and $p^{\delta} H_{k}=\sum_{i=1}^{k} C_{i}$ with $C_{i}=\sum_{\mu \in \Gamma_{i}}\left\langle v_{i}^{\mu}\right\rangle$ such that $O\left(v_{i}^{\mu}\right)=p^{i}$, for $k=n-1, n$. (The rank of $B_{n}$ is equal to the rank of $C_{n}$ since the rank of $G_{n} / G_{n-1}$ is equal to the rank of $H_{n} / H_{n-1}$.)

For each $\mu \in \Gamma_{n}$ there exists a sequence $\left\{x_{i}^{\mu}\right\}_{i \in \omega}$ of elements of $G_{n}$ satisfying the following conditions:

1. $x_{0}^{\mu}=u_{n}^{\mu}$

2. $p^{n i} x_{i}^{\mu}=x_{0}^{\mu}$ for all $i \in \omega$,

3. $x_{i}^{\mu}-p^{n} x_{i+1}^{\mu} \in G_{n-1}$ for all $i \in \omega$,

4. $G_{n} / G_{n-1}=\left\langle\left\{x_{i}^{\mu}+G_{n-1} \mid i \in \omega, \mu \in \Gamma_{n}\right\}\right\rangle$.

For each $\mu \in \Gamma_{n}$ we construct such a sequence inductively. Let $x_{0}^{\mu}=u_{n}^{\mu}$ and choose $x_{1}^{\mu} \in G_{n}$ such that $p^{n} x_{1}^{\mu}=x_{0}^{\mu}$. Assume that $x_{0}^{\mu}, \ldots, x_{i}^{\mu}$ have been obtained. Since $G_{n} / G_{n-1}$ is divisible there exists $y \in G_{n}$ such that $x_{i}^{\mu}+G_{n-1}=p^{n} y+G_{n-1}$. Thus $x_{0}^{\mu}+G_{n-1}=p^{n i} x_{i}^{\mu}+G_{n-1}=p^{n(i+1)} y+G_{n-1}$. Since $G_{n-1}$ is pure in $G_{n}$ and $h\left(x_{0}^{\mu}\right) \geqq \omega$, there exists $g \in G_{n-1}$ such that $x_{0}^{\mu}=p^{n(i+1)}(y+g)$. Let $x_{i+1}^{\mu}=y+g$. Then $p^{n(i+1)} x_{i+1}^{\mu}$ $=x_{0}^{\mu}$ and $x_{i}^{\mu}-p^{n} x_{i+1}^{\mu}=\left(x_{i}^{\mu}-p^{n} y\right)-p^{n} g \in G_{n-1}$. Thus conditions $1-4$ are satisfied.

Next, for each $\mu \in \Gamma_{n}$, there exists a sequence $\left\{y_{i}^{\mu}\right\}_{i \in \omega}$ of elements of $H_{n}$ satisfying the following conditions:

$1^{\prime} . p^{\delta} H_{n}=\sum_{i=1}^{n-1} C_{i} \oplus \sum_{\mu \in \Gamma_{n}}\left\langle y_{0}^{\mu}\right\rangle$ such that $O\left(y_{0}^{\mu}\right)=p^{n}$,

$2^{\prime} . p^{n i} y_{i}^{\mu}=y_{0}^{\mu}$ for all $i \in \omega$,

3'. $y_{i}^{\mu} \in \sigma\left(x_{i}^{\mu}+p^{\delta} G_{n}\right)$,

$4^{\prime} . y_{i}^{\mu}-p^{n} y_{i+1}^{\mu}=\alpha\left(x_{i}^{\mu}-p^{n} x_{i+1}^{\mu}\right) \in H_{n-1}$,

5'. $H_{n} / H_{n-1}=\left\langle\left\{y_{i}^{\mu}+H_{n-1} \mid i \in \omega, \mu \in \Gamma_{n}\right\}\right\rangle$.

Let $\mu \in \Gamma_{n}$. Choose $w_{i}^{\mu} \in \sigma\left(x_{i}^{\mu}+p^{\delta} G_{n}\right)$ for each $i \in \omega^{*}$. Note that

$$
O\left(w_{i}^{\mu}+p^{\delta} H_{n}\right)=O\left(\sigma^{-1}\left(w_{i}^{\mu}+p^{\delta} H_{n}\right)\right)=O\left(x_{i}^{\mu}+p^{\delta} G_{n}\right)=p^{n i} .
$$

Thus $p^{n i} \leqq O\left(w_{i}^{\mu}\right) \leqq p^{n(i+1)}$. Then

$$
p^{n i} w_{i}^{\mu}=k+p^{s} \sum_{\mu \in \Gamma_{n}} a_{\mu} v_{n}^{\mu}
$$

such that $k \in p^{\delta} H_{n-1}, 0 \leqq s \leqq n, a_{\mu}=0$ for almost all $\mu \in \Gamma_{n}$, and there exists $\eta \in \Gamma_{n}$ such that $0<a_{\eta}<p$. Suppose $s>0$. Let $k^{\prime} \in H_{n-1}$ such that $p k^{\prime}=k$. Then

$$
p^{n i-1} w_{i}^{\mu}-p^{s-1} \sum_{\mu \in \Gamma_{n}} a_{\mu} v_{n}^{\mu}+k^{\prime}=h+m,
$$

$h \in\left(H_{n-1}\right)[p]$ and $m \in C_{n}[p]$. Thus

$$
p^{n i-1} x_{i}^{\mu}+p^{\delta} G_{n}=\sigma^{-1}\left(p^{n i-1} w_{i}^{\mu}+p^{\delta} H_{n}\right)=\alpha^{-1}\left(h-k^{\prime}\right)+p^{\delta} G_{n} .
$$

Therefore $p^{n i-1} x_{i}^{\mu}=\alpha^{-1}\left(h-k^{\prime}\right)+g$ for some $g \in p^{\delta} G_{n}$. This implies that $\alpha^{-1}(-k)+p g$ $=p^{n i} x_{i}^{\mu}=x_{0}^{\mu}$ which is a contradiction. Thus $O\left(w_{i}^{\mu}\right)=p^{n(i+1)}$.

Let $y_{0}^{\mu}=p^{n} w_{1}^{\mu}$ for each $\mu \in \Gamma_{n}$. Then $\sum_{\mu \in \Gamma_{n}}\left\langle y_{0}^{\mu}\right\rangle$ is direct and

$$
p^{\delta} H_{n}=\sum_{i=1}^{n-1} C_{i} \oplus \sum_{\mu \in \Gamma_{n}}\left\langle y_{0}^{\mu}\right\rangle .
$$


To show that the sum is direct, first note that any $y \in\left\langle\left\{y_{0}^{\mu} \mid \mu \in \Gamma_{n}\right\}\right\rangle$ may be expressed as $y=p^{s} \sum_{\mu \in \Gamma_{n}} a_{\mu} y_{0}^{\mu}$, where $0 \leqq s \leqq n, 0 \leqq a_{\mu}<p^{n}$ for all $\mu \in \Gamma_{n}, a_{\mu}=0$ for almost all $\mu \in \Gamma_{n}$, and $0<a_{\eta}<p$ for some $\eta \in \Gamma_{n}$. Suppose that $0=p^{s} \sum_{\mu \in \Gamma_{n}} a_{\mu} y_{0}^{\mu}$ with $s<n$. Then from Lemma 1.1 we have $p^{n-1} \sum_{\mu \in \Gamma_{n}} a_{\mu} w_{1}^{\mu}=h+k$ such that $h \in\left(H_{n-1}\right)\left[p^{s+1}\right]$ and $k \in C_{n}\left[p^{s+1}\right]$. Thus

$$
p^{n-1} \sum_{\mu \in \Gamma_{n}} a_{\mu} x_{1}^{\mu}+p^{\delta} G_{n}=\sigma^{-1}\left(p^{n-1} \sum_{\mu \in \Gamma_{n}} a_{\mu} w_{1}^{\mu}+p^{\delta} H_{n}\right)=\alpha^{-1}(h)+p^{\delta} G_{n} .
$$

Hence $p^{n+s} \sum_{\mu \in \Gamma_{n}} a_{\mu} x_{1}^{\mu}=p^{s+1} \alpha^{-1}(h)+p^{s+1} g=p^{s+1} g$ for some $g \in p^{\delta} G_{n}$. But the height of $p^{n+s} \sum_{\mu \in \Gamma_{n}} a_{\mu} x_{1}^{\mu}$ in $p^{\downarrow} G_{n}$ is $s$ (since $0<a_{\eta}<p$ ) which is a contradiction. Thus the sum is direct. That $p^{\delta} H_{n}=\sum_{i=1}^{n-1} C_{i} \oplus \sum_{\mu \in \Gamma_{n}}\left\langle y_{0}^{\mu}\right\rangle$ follows from the fact that $\sigma$ is an isomorphism onto $H_{n} / p^{\delta} H_{n-1}$.

Assume that $y_{0}^{\mu}, \ldots, y_{i-1}^{\mu}$ have been defined such that conditions $2^{\prime}, 3^{\prime}$, and $4^{\prime}$ are satisfied, $y_{j}^{\mu}-w_{j}^{\mu} \in p^{\delta} G_{n}$ for $0<j<i$, and $\alpha\left(x_{i-1}^{\mu}-p^{n} x_{i}^{\mu}\right)=y_{i-1}^{\mu}-p^{n} w_{i}^{\mu}$. Choose $y_{i}^{\mu}=w_{i}^{\mu}-a$ where $a=w_{i}^{\mu}-p^{n} w_{i+1}^{\mu}-\alpha\left(x_{i}^{\mu}-p^{n} x_{i+1}^{\mu}\right) \in p^{\delta} G_{n}$. It is easily seen that the sequences $\left\{y_{i}^{\mu}\right\}_{i \in \omega}, \mu \in \Gamma_{n}$, satisfy conditions $1^{\prime}-5^{\prime}$.

With the sequence just constructed we can extend $\alpha$ to an isomorphism from $G_{n}$ onto $H_{n}$. First we note that if $x \in G_{n}$, then $x$ can be expressed uniquely as $x=c+\sum_{\mu \in \Gamma_{n}} \sum_{i \in \omega} a_{i}^{\mu} x_{i}^{\mu}$ where $0 \leqq a_{i}^{\mu}<p^{n}, c \in G_{n-1}$, and $a_{i}^{\mu}=0$ for almost all $i \in \omega$ and $\mu \in \Gamma_{n}$. This follows from Theorem 29.7 in [4]. Thus the desired extension is obtained by defining

$$
\begin{aligned}
\beta: G_{n} \rightarrow H_{n}: \beta(x) & =\beta\left(c+\sum_{\mu \in \Gamma_{n}} \sum_{i \in \omega} a_{i}^{\mu} x_{i}^{\mu}\right) \\
& =\alpha(c)+\sum_{\mu \in \Gamma_{n}} \sum_{i \in \omega} a_{i}^{\mu} y_{i}^{\mu} .
\end{aligned}
$$

It is clear that $\beta$ is a well-defined, one-to-one, onto map. Let $x, y \in G_{n}$. Then $x=c+\sum_{\mu \in \Gamma_{n}} \sum_{i \in \omega} a_{i}^{\mu} x_{i}^{\mu}$ and $y=d+\sum_{\mu \in \Gamma_{n}} \sum_{i \in \omega} b_{i}^{\mu} x_{i}^{\mu}$ where $c, d \in G_{n-1}$ and $a_{i}^{\mu}, b_{i}^{\mu}$ are restricted as above. In order to consider the sum $x+y$ we develop the following. Let $\mu \in \Gamma_{n}$. If $a_{i}^{\mu}, b_{i}^{\mu}=0$ for all $i \in \omega$ define $c_{i}^{\mu}=0$ for all $i \in \omega$. If not, let $j$ be the largest integer such that $a_{j}^{\mu}$ or $b_{j}^{\mu} \neq 0$. There exists unique integers $m_{j}$ and $r_{j}$ such that $a_{j}+b_{j}=m_{j} p^{n}+r_{j}$ with $0 \leqq r_{j}<p^{n}\left(m_{j}=0,1\right)$. Assuming $m_{j-i+1}, r_{j-i+1}$ have been obtained for $i \leqq j$, let $r_{j-i}$ and $m_{j-i}$ be the unique integers such that $a_{j-i}+b_{j-i}$ $+m_{j-i+1}=m_{j-i} p^{n}+r_{j-i}$ with $0 \leqq r_{j-i}<p^{n}\left(m_{j-i}=0,1\right)$. Let $c_{i}^{\mu}=r_{i}$ for $i=0, \ldots, j$ and $c_{i}^{\mu}=0$ for $i>j$. If $m_{j-i} \neq 0,0 \leqq i<j$, let $d_{i}^{\mu}=p^{n} x_{j-i}^{\mu}-x_{j-i-1}^{\mu}$. Otherwise let $d_{i}^{\mu}=0$. Then $x+y=c+d+\sum_{\mu \in \Gamma_{n}} \sum_{i \in \omega}\left(c_{i}^{\mu} x_{i}^{\mu}+d_{i}^{\mu}\right)$ where $d_{i}^{\mu} \in G_{n-1}$. Thus

$$
\beta(x+y)=\alpha(c+d)+\sum_{\mu \in \Gamma_{n}} \sum_{i \in \omega}\left(c_{i}^{\mu} y_{i}^{\mu}+\alpha\left(d_{i}^{\mu}\right)\right)=\beta(x)+\beta(y)
$$

where the second equality follows from condition $4^{\prime}$. Therefore $\beta$ is the desired isomorphism.

The following lemma will be useful in determining how the Ulm factors are put together to form the group. 
Lemma 1.3. Let $G$ be a reduced p-group, $\delta$ a limit ordinal, and $\left\{G_{i}\right\}_{i \in \omega}$ a chain $\left(G_{i} \subset G_{i+1}\right)$ of $(\delta+i)$-high subgroups of $G$. Let $G_{\delta}=\bigcup_{i \in \omega} G_{i}$. Then $G=\left\langle G_{\delta}, p^{\delta} G\right\rangle$. $p^{\delta} G=\bigcup_{i \in \omega} p^{\delta} G_{i}$ if and only if $G=\bigcup_{i \in \omega} G_{i}$. Also $p^{\delta} G_{\delta}$ is a basic subgroup of $p^{\delta} G$.

Proof. Since $G_{n}$ is isotype (see [8]), $p^{\delta} G_{n}=\sum_{i=1}^{n} B_{i}$, such that $B_{i}$ is a direct sum of cyclic groups of order $p^{i} ; B=\sum_{i \in \omega} B_{i}$ is a basic subgroup of $p^{\delta} G$; and $p^{\delta} G=$ $\sum_{i=1}^{n} B_{i} \oplus K_{n}$ where $K_{n}=\left\langle\sum_{i=n+1}^{\infty} B_{i}, p^{\delta+n} G\right\rangle$. Let $x \in G$ with order $p^{k+1}$. By Lemma $1.1, x=a+b$ with $a \in G_{k}\left[p^{k+1}\right]$ and $b \in K_{k}\left[p^{k+1}\right]$. Thus $x \in\left\langle G_{\delta}, p^{\delta} G\right\rangle$. The lemma follows.

CoRollary 1.4. $G=\left\langle G_{\delta}, p^{\delta+n} G\right\rangle, n$ an integer.

COROllaRy 1.5. $G / p^{\delta} G \cong G_{\delta} / p^{\delta} G_{\delta}$.

Proof. $G / p^{\delta} G=\left(G_{\delta}+p^{\delta} G\right) / p^{\delta} G \cong G_{\delta} /\left(G_{\delta} \cap p^{\delta} G\right)=G_{\delta} / p^{\delta} G_{\delta} .\left(G_{\delta} \cap p^{\delta} G=p^{\delta} G_{\delta}\right.$ since $G_{\delta}$ is isotype.)

COROLlaRY 1.6. Using the notation of Lemma 1.3 suppose there exists isomorphisms $\alpha$ from $G_{\delta}$ onto $H_{\delta}$ and $\beta$ from $p^{\delta} G$ onto $p^{\delta} H$ such that $\alpha\left|p^{\delta} G_{\delta}=\beta\right| p^{\delta} G_{\delta}$. Then there exists an (unique) isomorphism $\varphi$ from $G$ onto $H$ such that $\varphi \mid G_{\delta}=\alpha$ and $\varphi \mid p^{\delta} G=\beta$.

Proof. If $g \in G$ then $g=a+b$ with $a \in G_{\delta}$ and $b \in p^{\delta} G$. Thus define

$$
\varphi: G \rightarrow H: \varphi(g)=\alpha(a)+\beta(b) .
$$

It is easily seen that $\varphi$ is an isomorphism.

THEOREM 1.7. Let $G$ and $H$ be reduced p-groups and $\delta$ a limit ordinal such that $p^{\delta} G$ and $p^{\delta} H$ are torsion complete. A necessary and sufficient condition for $G$ and $H$ to be isomorphic is that there exist sequences $\left\{G_{i}\right\}_{i \in \omega}$ and $\left\{H_{i}\right\}_{i \in \omega}$ of subgroups of $G$ and $H$, respectively, satisfying the following conditions:

1. For each $i \in \omega, G_{i}$ and $H_{i}$ are $(\delta+i)$-high subgroups of $G$ and $H$, respectively.

2. For each $i \in \omega, G_{i} \subset G_{i+1}$ and $H_{i} \subset H_{i+1}$.

3. There exists a sequence $\left\{\varphi_{i}\right\}_{i \in \omega}$ such that $\varphi_{i}$ is an isomorphism from $G_{i} / p^{\delta} G_{i}$ onto $H_{i} / p^{\delta} H_{i}$ such that if $i>0, \varphi_{i} \mid G_{i-1} / p^{\delta} G_{i-1}=\varphi_{i-1}$ where $G_{i-1} / p^{\delta} G_{i-1}$ is embedded in $G_{i} / p^{\delta} G_{i}$ in the natural manner.

4. $p^{\delta} G$ and $p^{\delta} H$ are isomorphic.

Proof. The necessity is clear. For the sufficiency we first construct a sequence $\left\{\hat{\varphi}_{i}\right\}_{i \in \omega}$ such that $\hat{\varphi}_{i}$ is an isomorphism from $G_{i}$ onto $H_{i}, \hat{\varphi}_{i} \mid G_{i-1}=\hat{\varphi}_{i-1}, i>0$, and $\varphi_{i}\left(x+p^{\delta} G_{i}\right)=\hat{\varphi}_{i}(x)+p^{\delta} H_{i}$ for all $x \in G_{i}$. We proceed by induction. Let $\hat{\varphi}_{0}=\varphi_{0}$. Assuming that $\hat{\varphi}_{i-1}$ has been constructed, note that $\varphi_{i}\left(x+p^{\delta} G_{i}\right)=\hat{\varphi}_{i-1}(x)+p^{\delta} H_{i}$ for all $x \in G_{i-1}$. Thus by Theorem 1.2 there exists an isomorphism $\hat{\varphi}_{i}$ from $G_{i}$ onto $H_{i}$ such that $\varphi_{i}\left(x+p^{\delta} G_{i}\right)=\hat{\varphi}_{i}(x)+p^{\delta} H_{i}$ and $\hat{\varphi}_{i} \mid G_{i-1}=\hat{\varphi}_{i-1}$. Let $G_{\delta}=\bigcup_{i \in \omega} G_{i}$ and $H_{\delta}=\bigcup_{i \in \omega} H_{i}$. Let $\varphi$ be an isomorphism from $G_{\delta}$ onto $H_{\delta}$ such that $\varphi \mid G_{i}=\hat{\varphi}_{i}$. Let $\beta$ be an isomorphism from $p^{\delta} G$ onto $p^{\delta} H$ such that $\beta\left|p^{\delta} G_{\delta}=\varphi\right| p^{\delta} G_{\delta}$. ( $\beta$ exists since 
$p^{\delta} G$ is torsion complete and $p^{\delta} G_{\delta}$ is a basic subgroup of $p^{\delta} G$ (see [11]).) By Corollary 1.6, there exists an isomorphism $\gamma$ from $G$ onto $H$ such that $\gamma \mid G_{\delta}=\varphi$ and $\gamma \mid p^{\delta} G=\beta$. Thus $G$ and $H$ are isomorphic.

If we define the chain of subgroups $\left\{\left(G_{i}+p^{\delta} G\right) / p^{\delta} G\right\}_{i \in \omega}$ to be a " $\delta$-high chain" in $G / p^{\delta} G$ then, in the case that $p^{\delta} G$ is torsion complete, $G$ is characterized by its class of " $\delta$-high chains" and $p^{\delta} G$.

CoRollary 1.8. Let $G$ and $H$ be p-groups such that $G / p^{\omega} G \cong H / p^{\omega} H, G / p^{\omega} G$ is torsion complete, $p^{\omega} G \cong p^{\omega} H \cong \sum Z\left(p^{n}\right)$ ( $n$ fixed), and $G$ and $H$ have isomorphic high subgroups. Then $G$ and $H$ are isomorphic.

Under the conditions of Corollary 1.8 we have that $G$ is characterized by $G / p^{\omega} G$, $p^{\omega} G$, and its class of high subgroups.

LEMMA 1.9. Let $G$ be a reduced p-group, $H$ a subgroup of $G$ with basic subgroup $B$, and $\alpha$ an ordinal. If $B \subset p^{\alpha} G$ then $H \subset p^{\alpha} G$.

Proof. Assume that $H \subset p^{\beta} G$ for all $\beta<\sigma \leqq \alpha$. Let $x \in H$. If $\sigma$ is not a limit ordinal let $\delta$ be such that $\sigma=\delta+1$. Then $x \in p^{\delta} G$, and since $B$ is basic in $H$ there exists $y \in H \subset p^{\delta} G$ such that $p y-x=b \in B$. Since $B \subset p^{\alpha} G \subset p^{\sigma} G$ there exists $z \in p^{\delta} G$ such that $p z=b$. Thus $p(y-z)=x$, and $x \in p^{\sigma} G$. If $\sigma$ is a limit ordinal then $x \in p^{\beta} G$ for all $\beta<\sigma$ and hence $x \in p^{\sigma} G$. Thus $H \subset p^{\sigma} G$. Therefore $H \subset p^{\alpha} G$.

LEMMA 1.10. Let $G$ be a reduced p-group and let $\left\{\alpha_{j}\right\}_{j \in \omega}$ be an increasing sequence of ordinals such that $\alpha_{0}=0$. For each $j \in \omega$, let $\beta_{j}$ be the ordinal such that $\omega \alpha_{j}+\beta_{j}$ $=\omega \alpha_{j+1}$. For each $j \in \omega$ let $\left\{K_{i}^{j}\right\}_{i \in \omega}$ be a chain of subgroups of $G$ such that $K_{i}^{j}$ is $\left(\beta_{j}+1\right)$-high in $p^{\omega \alpha_{j} G}$, and $K_{0}^{j+1} \supset p^{\beta_{j} K_{\omega}^{j}}$ where $K_{\omega}^{j}=\bigcup_{i \in \omega} K_{i}^{j}$. Then $S_{i}^{n}=$ $\left\langle\left\{K_{\omega}^{j} \mid j<n\right\}, K_{i}^{n}\right\rangle$ is $\left(\omega \alpha_{n+1}+i\right)$-high in $G$.

Proof. Well order $\{(n, i) \mid n, i \in \omega\}$ by $(n, i)<(m, k)$ if $n<m$ and $(n, i)<(m, k)$ if $n=m$ and $i<k$. If $n=0$ then clearly $S_{i}^{0}$ is $(\omega+i)$-high in $G$ for all $i \in \omega$. Suppose the result is true for all $(n, i)<(m, k), m>0$. Note that $S_{k}^{m} \cap p^{\omega \alpha_{m+1}+k} G=0$, and let $S$ be an $\left(\omega \alpha_{m+1}+k\right)$-high subgroup of $G$ containing $S_{k}^{m}$. Let $x \in S$ and suppose $O(x)=p^{r+1}$ for some $r \in \omega$. First note that by Lemma 1.9 and the induction hypothesis we have $p^{\omega \alpha_{m}} S_{k}^{m}=K_{k}^{m}$. Next note that $S_{k}^{m}\left[p^{r+1}\right]=S_{r}^{m-1}\left[p^{r+1}\right] \oplus M\left[p^{r+1}\right]$ where $M$ is a complementary summand of a maximal $p^{r}$-bounded summand of $K_{k}^{m}$. This follows from the fact that $p^{\omega \alpha_{m}} S_{k}^{m}=K_{k}^{m}$ and Lemma 1.1. Let $B$ be a basic subgroup of $M$ and $L=\left\langle B, p^{\omega \alpha_{m}+r} G\right\rangle$. Then $M \subset L$ and $G\left[p^{r+1}\right]=S_{r}^{m-1}\left[p^{r+1}\right]$ $\oplus L\left[p^{r+1}\right]$. (Again the induction hypothesis and Lemma 1.1 are used.) Now $x=a+b$ such that $a \in S_{r}^{m-1}\left[p^{r+1}\right]$ and $b \in L\left[p^{r+1}\right]$. Thus $b \in S \cap p^{\omega \alpha_{m} G}$ and it follows that $\left\langle b, K_{k}^{m}\right\rangle \cap p^{\omega \alpha_{m+1}+k} G=0$. Since $K_{k}^{m}$ is $\left(\beta_{m}+k\right)$-high in $p^{\omega \alpha_{m}} G, b \in K_{k}^{m}$. Therefore $S=S_{k}^{m}$.

COROLlaRY 1.11. $S_{i}^{n}$ is isotype in $G$. 
COROLlaRY 1.12. Following the notation of Lemma 1.10, the map

$$
\sigma: S_{k}^{m} / p^{\omega \alpha_{m}} S_{k}^{m} \rightarrow G / p^{\omega \alpha_{m} G}: x+p^{\omega \alpha_{m}} S_{k}^{m} \rightarrow x+p^{\omega \alpha_{m} G}
$$

is an isomorphism of $S_{k}^{m} / p^{\omega \alpha_{m}}$ onto $G / p^{\omega \alpha_{m} G}$.

Proof. Let $x \in G$ such that $O(x)=p^{r+1}$. Then $G\left[p^{r+1}\right]=S_{r}^{m-1}\left[p^{r+1}\right] \oplus L\left[p^{r+1}\right]$ and $x=a+b$ with $a \in S_{r}^{m-1}\left[p^{r+1}\right]$ and $b \in L\left[p^{r+1}\right]$. Since $L \subset p^{\omega \alpha_{m} G, \sigma\left(a+p^{\omega \alpha_{m}} S_{k}^{m}\right)}$ $=x+p^{\omega \alpha_{m} G}$. Hence $\sigma$ is onto. The result follows easily.

COROLlary 1.13. Following the notation of Lemma 1.10,

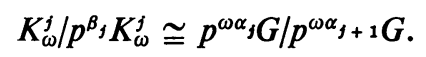

REMARK 1.14 (TopologiCal CONSIDERATIONS). Let $G$ be a reduced $p$-group, $\beta$ an ordinal $\geqq 1$. Let $\mathscr{T}^{\beta}$ be the topology induced on $G$ by taking the chain of subgroups $\left\{p^{\alpha} G\right\}_{\alpha \in \omega \beta}$ as a fundamental system of neighborhoods of 0 . Let $\mathscr{T}_{n}$ be the relative topology induced on $G\left[p^{n}\right]$ by $\mathscr{T}^{\beta}$. Let $\mathscr{T}_{\beta}$ be the inductive limit topology on $G$ obtained by taking the inductive limit of the topological groups $\left(G\left[p^{n}\right], \mathscr{T}_{n}\right)$ in the category of topological abelian groups (see [2]). We shall refer to $\mathscr{T}_{\beta}$ as the $\beta$-topology on $G$. Note that $\mathscr{T}_{\beta}$ is Hausdorff if the Ulm type of $G$ is $\leqq \beta$, and, in this case, the completion of $G$ with respect to the $\beta$-topology is the torsion subgroup of the completion of $G$ with respect to $\mathscr{T}^{\beta}$.

To obtain a convenient fundamental system of neighborhoods of zero for the $\beta$-topology we need a special class of I. Kaplansky's $U$-sequences (see [10]). Let $G$ be a reduced $p$-group of Ulm type $\geqq \lambda$ ( $\lambda$ a limit ordinal). A sequence $\tilde{\alpha}=\left\{\alpha_{i}\right\}_{i \in \omega}$ with $\alpha_{0}, \alpha_{1}, \alpha_{2}, \ldots$ ordinals less than $\omega \lambda$ is called a $(\lambda-U)$-sequence for $G$ if

(i) $\alpha_{0}<\alpha_{1}<\alpha_{2}<\cdots$, and

(ii) $\alpha_{k}+1<\alpha_{k+1}$ implies $f_{G}\left(d_{k}\right) \neq 0$ where $f_{G}\left(\alpha_{k}\right)$ is the $\alpha_{k}$ th Ulm invariant of $G$. The Ulm sequence of an element $x \in G$ is defined to be $U_{G}(x)=\left\{h_{G}\left(p^{i} x\right)\right\}_{i \in \omega}$ (the height of $p^{i} x$ in $G$ ).

Partially order these sequences componentwise. If $\tilde{\alpha}$ is a $(\lambda-U)$-sequence for $G$, then $G\{\tilde{\alpha}\}=\left\{x \in G \mid U_{G}(x) \geqq \tilde{\alpha}\right\}$ is an unbounded fully invariant subgroup of $G$. Let $K$ be the set of all $(\lambda-U)$-sequences for $G$. Let $\mathscr{F}=\{G\{\tilde{\alpha}\} \mid \tilde{\alpha} \in K\}$. Then $\mathscr{F}$ is a fundamental system of neighborhoods of zero for $\mathscr{T}_{\beta}$.

REMARK 1.15 (SUBDIRECT SUM). Let $G$ be an unbounded reduced $p$-group of Ulm type $\beta \geqq 1$. If $G$ has Ulm type 1 we endow $G$ with the large topology (see [3]). If $G$ has Ulm type $\beta$ we endow $G$ with the $\beta$-topology. Let $H$ be a dense isotype subgroup of $G$; let $B$ be a direct sum of cyclic $p$-groups such that $B$ has the same rank as $G / H$; let $D$ be a divisible hull of $B$; let $\sigma: G \rightarrow G / H$ and $\gamma: D \rightarrow D / B$ be the natural homomorphisms; and let $f$ be an isomorphism from $G / H$ onto $D / B$. Define $K=\{(g, d) \mid g \in G, d \in D$, and $f(\sigma(g))=\gamma(d)\}$, a subgroup of $G \oplus D$. It can be shown that $H \oplus\{0\}$ is an $\omega \beta$-high subgroup of $K$ and $\{0\} \oplus B=p^{\omega \beta} K$. We shall identify $H$ with $H . \oplus\{0\}$ and $B$ with $\{0\} \oplus B$. We shall refer to $K$ as the subdirect sum of $G$ and $D$ with $\omega \beta$-high subgroup $H$ and $p^{\omega \beta} K=B$. 
Let $B=\sum_{i \in \omega} B_{i}$ with $B_{i}=\sum_{\alpha \in \Gamma_{i}}\left\langle x_{\alpha, i}\right\rangle$ and $O\left(x_{\alpha, i}\right)=p^{i}$ for all $\alpha \in \Gamma_{i}$, and let $\left\{H_{i}\right\}_{i \in \omega}$ be a chain of dense isotype subgroups of $G$ such that $\bigcup_{i \in \omega} H_{i}=G$ and $H_{i+1} / H_{i}$ has the same rank as $B_{i}$. Let $D_{i}$ be a divisible hull of $B_{i}$ for each $i \in \omega$, $D=\sum_{i \in \omega} D_{i}$, and choose a $y_{\alpha, i} \in D_{i}$, for each $\alpha \in \Gamma_{i}$ and $i \in \omega^{*}$, such that $p y_{\alpha, i}=x_{\alpha, i}$. Let $L_{i}=\sum_{\alpha \in \Gamma_{i}}\left\langle z_{\alpha, i}\right\rangle$ be a complementary summand of $H_{i}[p]$ in $H_{i+1}[p]$ for each $i \in \omega$. Let $\sigma: G \rightarrow G / H_{0}$ and $\mathscr{T}_{i}: D_{i} \rightarrow D_{i} / B_{i}$ for each $i \in \omega$ be natural homomorphisms, and let $f$ be an isomorphism from $G / H_{0}$ onto $\sum_{i \in \omega} D_{i} / B_{i}$ such that $f \mid H_{j} / H_{0}=\sum_{i=1}^{j} D_{i} / B_{i}$ and $f\left(z_{\alpha, i}+H_{0}\right)=y_{\alpha, i}+\sum B_{i}$. Define $K=\{(g, d) \mid g \in G, d \in D$, and $f(\sigma(g))=\sum \mathscr{T}_{i}\left(d_{i}\right)$ where $\left.d=\sum d_{i}, d \in D_{i}\right\}$, a subgroup of $G \oplus D$. It is easily shown that $H_{i}^{\prime}=\left\{(h, d) \mid h \in H_{i}, d \in \sum_{j=0}^{i} D_{j}\right.$, and $f(\sigma(h))=\sum \gamma_{j}\left(d_{j}\right)$ where $d=\sum d_{j}$, $\left.d_{j} \in D_{j}\right\}$ is $(\omega \beta+i)$-high in $K$ and $p^{\omega \beta} K=B$. In this case we shall refer to $K$ as the subdirect sum of $G$ and $D$ with " $\beta$-high chain" $\left\{H_{i}\right\}_{i \in \omega}$ and $p^{\omega \beta} K=B$.

REMARK 1.16. If $\alpha>\omega$ is a countable limit ordinal, then $\alpha$ is of one and only one of the following types:

Type 1. There exists a limit ordinal $\beta<\alpha$ such that $\beta \omega>\alpha$.

Type 2. There exists a sequence $\left\{\alpha_{i}\right\}_{i \in \omega}$ of countable limit ordinals such that $\alpha_{0}=0, \alpha_{i+2}-\alpha_{i+1} \geqq \alpha_{i+1}-\alpha_{i}$ for all $i \in \omega$, and $\lim _{i \in \omega} \alpha_{i}=\alpha$.

Note that if $\alpha$ is of type 1 we may choose $\beta$ of type 2, and if $\alpha$ is of type 2, we may choose the $\alpha_{i}$ 's of type 2 . Hence we will call a sequence $\left\{\alpha_{i}\right\}_{i \in \omega}$ of countable limit ordinals an admissible sequence if $\alpha_{0}=0, \alpha_{i+2}-\alpha_{i+1} \geqq \alpha_{i+1}-\alpha_{i}$ for all $i \in \omega$, and $\alpha_{i}$ is of type 2 for all $i \in \omega$.

2. Reduced $p$-primary abelian groups of Ulm type $\leqq \omega$. We will first consider groups of finite type and determine their structure in terms of the Ulm factors, sequences of subgroups in each Ulm factor, and sequences of sets of elements of each Ulm factor that determine how the Ulm factors were "connected." We will then define an $\omega$-summable group whose structure is determined in a similar manner, induce a topology on these groups, and show that every group of Ulm type $\omega$ is an isotype subgroup of the completion of an $\omega$-summable group.

Definition 2.1. Let $G$ be a reduced $p$-group and $\alpha$ an ordinal number such that $\alpha<\tau(G)$. Let $\left\{K_{i}\right\}_{i \in \omega}$ be a chain of subgroups of $p^{\omega \alpha} G$ such that $K_{i}$ is $(\omega+i)$-high in $p^{\omega \alpha} G$. Let $\sigma: p^{\omega \alpha} G \rightarrow G_{\alpha}=p^{\omega \alpha} G / p^{\omega(\alpha+1)} G$ be the natural homomorphism. Then $\left\{K_{i}\right\}_{i \in \omega}$ will be called a high chain of $p^{\omega \alpha} G$ and $\left\{\sigma\left(K_{i}\right)\right\}_{i \in \omega}$ will be called a high chain of $G_{\alpha}$.

Definition 2.2. Let $G$ and $H$ be reduced $p$-groups and let $G_{\alpha}, \alpha \in \tau(G)$, and $H_{\alpha}$, $\alpha \in \tau(H)$, be the $\alpha$ th Ulm factors of $G$ and $H$, respectively. Let $\varphi_{i}: G_{\alpha+i} \rightarrow H_{\alpha+i}$, $i \in \omega$, be (onto) isomorphisms. Then $\left\{\varphi_{i}\right\}_{i \in \omega}$ is said to be compatible if for every $j \in \omega$ there exist

(i) high chains $\left\{K_{i}^{j}\right\}_{i \in \omega}$ and $\left\{L_{i}^{j}\right\}_{i \in \omega}$ of $p^{\omega(\alpha+j)} G$ and $p^{\omega(\alpha+j)} H$, respectively,

(ii) decompositions $p^{\omega} K_{i}^{j}=\sum_{k=1}^{i} \sum_{\mu \in \Gamma_{(k, j)}}\left\langle x_{k}^{(\mu, j)}\right\rangle$ and $p^{\omega} L_{i}^{j}=\sum_{k=1}^{i} \sum_{\mu \in \Gamma_{(k, j}}\left\langle y_{k}^{(\mu, j)}\right\rangle$ for each $i \in \omega^{*}$, and

(iii) $\left\{\left\{u_{i}^{(\mu, j)} \mid \mu \in \Gamma_{(i, j)}\right\}\right\}_{i \in \omega^{*}}, u_{i}^{(\mu, j)} \in K_{i}^{j}$, and $\left\{\left\{v_{i}^{(\mu, j)} \mid \mu \in \Gamma_{(i, j)}\right\}\right\}_{i \in \omega^{*}}, v_{i}^{(\mu, j)} \in L_{i}^{j}$ satisfying the following conditions: 
(a) For all $j \in \omega$, if $K_{\omega}^{j}=\bigcup_{i \in \omega} K_{i}^{j}$ and $L_{\omega}^{j}=\bigcup_{i \in \omega} L_{i}^{j}$ then $p^{\omega} K_{\omega}^{j} \subset K_{0}^{j+1}$ and $p^{\omega} L_{\omega}^{j} \subset L_{0}^{j+1}$.

(b) For all $\mu \in \Gamma_{(i, j)}, i \in \omega^{*}$, and $j \in \omega, p^{i} u_{i}^{(\mu, j)}=x_{i}^{(\mu, j)}$ and $p^{i} v_{i}^{(\mu, j)}=y_{i}^{(\mu, j)}$.

(c) For all $\mu \in \Gamma_{(i, j)}, i \in \omega$, and $j \in \omega$, if $\sigma_{j}: p^{\omega(\alpha+j)} G \rightarrow G_{\alpha+j}$ and $\rho_{j}: p^{\omega(\alpha+j)} H \rightarrow$ $H_{\alpha+j}$ are the natural homomorphisms, then $\varphi_{j} \sigma_{j}\left(u_{i}^{(\mu, j)}\right)=\rho_{j}\left(v_{i}^{(\mu, j)}\right), \varphi_{j+1} \sigma_{j+1}\left(x_{i}^{(\mu, j)}\right)$ $=\rho_{j+1}\left(y_{i}^{(\mu, j)}\right)$, and $\varphi_{j} \sigma_{j}\left(K_{i}^{j}\right)=\rho_{j}\left(L_{i}^{j}\right)$.

We state the following structure theorem in spite of its inelegance because of its structural value.

THEOREM 2.3. Let $G$ and $H$ be reduced p-groups with at most a finite number of Ulm factors $G_{0}, G_{1}, \ldots, G_{m}$ and $H_{0}, H_{1}, \ldots, H_{m}$, respectively. $A$ necessary and sufficient condition for $G$ and $H$ to be isomorphic is that there exist isomorphisms $\varphi_{j}: G_{j} \rightarrow H_{j}, j=0, \ldots, m$, such that $\left\{\varphi_{j}\right\}_{j=0}^{m}$ is compatible.

Proof. Since the necessity is clear we will prove only the sufficiency. We will use the notation of Definition 2.2 with $\alpha=0$.

Let $\sigma: G \rightarrow G / p^{\omega 2} G$ and $\rho: H \rightarrow H / p^{\omega 2} H$ be the natural homomorphisms. First we construct an isomorphism $\alpha_{0}$ from $G / p^{\omega 2} G$ onto $H / p^{\omega 2} H$ such that $\alpha_{0} \sigma\left(u_{i}^{(\mu, 0)}\right)$ $=\rho\left(v_{i}^{(\mu, 0)}\right)$ for all $i \in \omega^{*}, \mu \in \Gamma_{(i, 0)}$. Construct, inductively on $n$, an isomorphism $\alpha$ from $\sigma\left(K_{\omega}^{0}\right)$ onto $\rho\left(L_{\omega}^{0}\right)$ such that in the $n$th step we use the construction in the proof of Theorem 1.2 and choose $x_{1}^{\mu}=\sigma\left(u_{n}^{(\mu, 0)}\right)$ and $w_{1}^{\mu}=\rho\left(v_{n}^{(\mu, 0)}\right)$ for all $\mu \in \Gamma_{(n, 0)}$. Thus $\alpha\left|\sigma\left(p^{\omega} K_{\omega}^{0}\right)=\varphi_{1}\right| \sigma\left(p^{\omega} K_{\omega}^{0}\right)$. By Corollary 1.6 there exists an isomorphism $\alpha_{0}$ from $G / p^{\omega 2} G$ onto $H / p^{\omega 2} H$ such that $\alpha_{0} \mid \sigma\left(K_{\omega}^{0}\right)=\alpha$ and $\alpha_{0} \mid G_{1}=\varphi_{1}$.

Next let $\sigma: G \rightarrow G / p^{\omega(k+2)} G$ and $\rho: H \rightarrow H / p^{\omega(k+2)} H$ be natural. Let

$$
S_{i}^{k}=\left\langle\left\{K_{\omega}^{j} \mid j<k\right\}, K_{i}^{k}\right\rangle \text { and } T_{i}^{k}=\left\langle\left\{L_{\omega}^{i} \mid j<k\right\}, L_{i}^{k}\right\rangle
$$

for all $i \in \omega$ and $S_{\omega}^{k}=\bigcup_{i \in \omega} S_{i}^{k}$ and $T_{\omega}^{k}=\bigcup_{i \in \omega} T_{i}^{k}$ (as considered in Lemma 1.10). Suppose an isomorphism $\alpha_{k}$ has been defined from $G / p^{\omega(k+1)} G$ onto $H / p^{\omega(k+1)} H$ such that $\alpha_{k} \mid G_{k}=\varphi_{k}$. Construct, inductively on $n$, an isomorphism $\alpha$ from $\sigma\left(S_{\omega}^{k}\right)$ onto $\rho\left(T_{\omega}^{k}\right)$ such that in the $n$th step we use the construction in the proof of Theorem 1.2 and choose $x_{1}^{\mu}=\sigma\left(u_{n}^{(\mu, k+1)}\right)$ and $w_{1}^{\mu}=\rho\left(v_{n}^{(\mu, k+1)}\right)$ for all $\mu \in \Gamma_{(n, k+1)}$. Thus $\alpha\left|\sigma\left(p^{\omega(k+1)} S_{\omega}^{k}\right)=\varphi_{k+1}\right| \sigma\left(p^{\omega(k+1)} S_{\omega}^{k}\right)$. By Corollary 1.6, there is an isomorphism $\alpha_{k+1}$ from $G / p^{\omega(k+2)}$ onto $H / p^{\omega(k+2)} H$ such that $\alpha_{k+1} \mid \sigma\left(S_{\omega}^{k}\right)=\alpha$ and $\alpha_{k+1} \mid G_{k+1}=\varphi_{k+1}$. Thus we obtain an isomorphism $\alpha_{m-1}$ from $G$ onto $H$.

COROLLARY 2.4. Any reduced p-group with a finite number of Ulm factors can be constructed from the Ulm factors using the methods of Theorem 38.1 in [4].

Definition 2.5. Let $G$ be a reduced $p$-group. Then $G$ will be said to be $\omega$-summable if there exists a chain of subgroups $\left\{G_{i}\right\}_{i \in \omega^{*}}$, with $G_{i}$ wi-high in $G$, such that $G=\bigcup_{i \in \omega^{*}} G_{i}$.

NotAtion 2.6. Let $\alpha$ and $\beta$ be limit ordinals, and $i, j \in \omega$. We shall call a subgroup $K_{j}^{i}$ of $G$ a $(\beta i, \alpha+j)$-subgroup of $G$ if $K_{j}^{i}$ is an $(\alpha+j)$-high subgroup of $p^{\beta i} G$. 
We shall call a subgroup $K_{\omega}^{i}$ of $G$ a $(\beta i,(\alpha, \omega))$-subgroup of $G$ if $K_{\omega}^{i}=\bigcup_{j \in \omega} K_{j}^{i}$ for some chain $\left\{K_{j}^{i}\right\}_{j \in \omega}$ of $(\beta i, \alpha+j)$-subgroups of $G$.

THEOREM 2.7. A reduced p-group $G$ is $\omega$-summable iff $G=\left\langle\left\{K_{\omega}^{i} \mid i \in \omega\right\}\right\rangle$ for some sequence $\left\{K_{\omega}^{i}\right\}_{i \in \omega}$ of $(\omega i,(\omega, \omega))$-subgroups of $G$ such that $p^{\omega} K_{\omega}^{i} \subset K_{\omega}^{i+1}$.

Proof. Clearly if $G=\left\langle\left\{K_{\omega}^{i} \mid i \in \omega\right\}\right\rangle$ we may take $G_{i}=\left\langle\left\{K_{\omega}^{k} \mid k<i-1\right\}, H\right\rangle$ where $H$ is a high subgroup of $K_{\omega}^{i-1}$ such that $H \supset p^{\omega} K_{\omega}^{i-2}$ if $i>1$. This follows from Lemma 1.10 .

If $G=\bigcup_{i \in \omega^{*}} G_{i}$ with $G_{i} \omega i$-high in $G$ we may construct a sequence $\left\{K_{\omega}^{i}\right\}_{i \in \omega}$ as follows. Let $K_{i}^{0}$ be a chain of $(\omega \cdot 0, \omega+i)$-subgroups of $G_{2}$ with $K_{0}^{0}=G_{1}$ and $K_{\omega}^{0}=\bigcup_{i \in \omega} K_{i}$. Assuming that $K_{\omega}^{n} \subset G_{n+2}$ has been constructed let $K_{0}^{n+1}$ be a $(\omega(n+1), \omega)$-subgroup of $G_{n+2}$ containing $p^{\omega} K_{\omega}^{n}$ and $\left\{K_{i}^{n+1}\right\}_{i \in \omega}$ be a chain of $(\omega(n+1), \omega+i)$-subgroups of $G_{n+3}$. Let $K_{\omega}^{n+1}=\bigcup_{i \in \omega} K_{i}^{n+1}$. Then it follows easily from Lemma 1.10 that $G=\left\langle\left\{K_{\omega}^{i} \mid i \in \omega\right\}\right\rangle$.

It follows that if $G$ is $\omega$-summable then $G \cong\left(\sum_{i \in \omega} K_{\omega}^{i}\right) / R$ where $K_{\omega}^{i}$ is a group of Ulm type $\leqq 2, p^{\omega} K_{\omega}^{i}$ is isomorphic to a basic subgroup of $K_{\omega}^{i+1}$, and $R$ is a relation which identifies $p^{\omega} K_{\omega}^{i}$ with a particular basic subgroup of $K_{\omega}^{i+1}$.

To construct $K_{\omega}^{i}$, let $\sigma_{i}: p^{\omega i} G \rightarrow p^{\omega i} G / p^{\omega(i+1)} G=G_{i}$, the $i$ th Ulm factor of $G$, be the natural homomorphism and $\left\{H_{\imath}\right\}_{i \in \omega}$ be a high chain of $p^{\omega i} G$. Let $B_{i+1}$ be a basic subgroup of $G_{i+1}$ and $D_{i+1}$ a divisible hull of $B_{i+1}$. Then $K_{\omega}^{i}$ can be constructed (up to isomorphism) as the subdirect sum of $G_{i}$ and $D_{i+1}$ with high chain $\left\{\sigma_{i}\left(H_{i}\right)\right\}_{i \in \omega}$ and elements of infinite height $B_{i+1}$. (The set $\left\{z_{\alpha, i} \mid \alpha \in \Gamma_{i}, i \in \omega\right\}$ as defined in Remark 1.15 should be carefully chosen.)

THEOREM 2.8. Let $G$ and $H$ be $\omega$-summable groups and let $G_{j}$ and $H_{j}$ be the jth Ulm factors of $G$ and $H$, respectively. A necessary and sufficient condition for $G$ and $H$ to be isomorphic is that there exist isomorphisms $\varphi_{j}: G_{j} \rightarrow H_{j}$ such that $\left\{\varphi_{j}\right\}_{j \in \omega}$ is compatible and the high chains in (i) of Definition 2.2 can be chosen such that they generate $G$ and $H$, respectively.

3. Reduced $p$-primary abelian groups of countable Ulm type. We shall need the following generalization of Ulm factor. If $G$ is a reduced $p$-group and $\beta$ is a limit ordinal such that $\beta=\omega$ or $\beta$ is of type 2 , then the $\alpha$ th $\beta$-factor of $G$ is defined to be $p^{\beta \alpha} G / p^{\beta(\alpha+1)} G$. Note that the $\alpha$ th $\omega$-factor is just the $\alpha$ th Ulm factor. Also if $\beta$ is the Ulm type of $G$ and $\left\{\alpha_{i}\right\}_{i \in \omega}$ is an admissible sequence converging to $\beta$, then we will

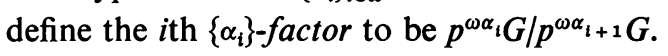

In this section we will determine the structure of a reduced $p$-primary abelian group of countable Ulm type in terms of the structure of its various factors.

Definition 3.1. Let $G$ be a reduced $p$-group, let $\beta$ be a (countable) limit ordinal of type 2, and let $\left\{\alpha_{i}\right\}_{i \in \omega}$ be an admissible sequence converging to $\beta$. Then $G$ will be said to be $\beta$-summable if there exists a chain of subgroups $\left\{G_{i}\right\}_{i \in \omega^{*}}$ with $G_{i} \omega \alpha_{i}$-high in $G$ such that $G=\bigcup_{i \in \omega^{*}} G_{i}$. A subgroup $C$ of $G$ is said to be a $\beta$-summable subgroup 
of $G$ if there exists a chain of subgroups $\left\{G_{i}\right\}_{i \in \omega^{*}}$ of $G$ with $G_{i} \omega \alpha_{i}$-high in $G$ such that $C=\bigcup_{i \in \omega^{*}} G_{i}$.

Note that the definition of a $\beta$-summable group is independent of the choice of the admissible sequence converging to $\beta$.

The next theorem is an immediate generalization of Theorem 2.5. Let $\alpha$ and $\beta$ be limit ordinals, $\left\{\alpha_{i}\right\}_{i \in \omega}$ a sequence of limit ordinals, and $j \in \omega$. Following the notation of the last section we call a subgroup $K_{j}^{i}$ of $G$ a $\left(\beta \alpha_{i}, \alpha+j\right)$-subgroup of $G$ if $K_{j}^{i}$ is an $(\alpha+j)$-high subgroup of $p^{\beta \alpha_{i}} G$ and a subgroup $K_{\omega}^{i}$ of $G$ a $\left(\beta \alpha_{i},(\alpha, \omega)\right)$-subgroup of $G$ if $K_{\omega}^{i}=\bigcup_{j \in \omega} K_{j}^{i}$ for some chain $\left\{K_{j}^{i}\right\}_{j \in \omega}$ of $\left(\beta \alpha_{i}, \alpha+j\right)$-subgroups of $G$.

THEOREM 3.2. Let $\beta$ be a countable limit ordinal of type 2. A reduced p-group $G$ is $\beta$-summable iff there exists an admissible sequence $\left\{\alpha_{i}\right\}_{i \in \omega}$ of limit ordinals converging to $\beta$ and a sequence $\left\{K_{\omega}^{i}\right\}_{i \in \omega}$ of $\left(\omega \alpha_{i},\left(\omega\left(\alpha_{i+1}-\alpha_{i}\right), \omega\right)\right)$-subgroups of $G$ with $p^{\omega\left(\alpha_{i+1}-\alpha_{i}\right)} K_{\omega}^{i} \subset K_{\omega}^{i+1}$ such that $G=\left\langle\left\{K_{\omega}^{i} \mid i \in \omega\right\}\right\rangle$.

The proof is similar to that of Theorem 2.5.

It follows that, if $G$ is $\beta$-summable, then $G \cong\left(\sum_{i \in \omega} K_{\omega}^{i}\right) / R$ where $K_{\omega}^{i}$ is a group of type $\leqq\left(\alpha_{i+1}-\alpha_{i}\right)+1$ and $R$ is a relation which identifies $p^{\omega} K_{\omega}^{i}$ with a particular basic subgroup of $K_{\omega}^{i+1}$.

To construct $K_{\omega}^{i}$, let $\sigma_{i}: p^{\omega \alpha_{i}} G \rightarrow p^{\omega \alpha_{i}} G / p^{\omega\left(\alpha_{i+1}-\alpha_{i}\right)} G=G_{i}$, the $i$ th $\left\{\alpha_{i}\right\}$-factor, be the natural homomorphism and $\left\{H_{i}\right\}_{i \in \omega}$ be a chain of subgroups of $p^{\omega \alpha_{i} G}$ such that $H_{i}$ is $\left(\omega\left(\alpha_{i+1}-\alpha_{i}\right)+i\right)$-high in $p^{\omega \alpha_{i}} G$. Let $B_{i+1}$ be a basic subgroup of $G_{i+1}$ and $D_{i+1}$ a divisible hull of $B_{i+1}$. Then $K_{\omega}^{i}$ can be constructed (up to isomorphism) as the subdirect sum of $G_{i}$ and $D_{i+1}$ with $\omega\left(\alpha_{i+1}-\alpha_{i}\right)$-high chain $\left\{\sigma_{i}\left(H_{i}\right)\right\}_{i \in \omega}$ and $p^{\omega\left(\alpha_{i+1}-\alpha_{i}\right)} K_{\omega}^{i}=B_{i+1}$. (Again the set $\left\{z_{\alpha, i} \mid \alpha \in \Gamma_{i}, i \in \omega\right\}$ as defined in Remark 1.15 should be carefully chosen.) (That $\sigma_{i}\left(H_{i}\right)$ is isotype and dense in the $\left(\alpha_{i+1}-\alpha_{i}\right)$ topology follows from Lemma 1.1 and results of [8]. The proofs are similar to those in [3].)

REMARK 3.3. Let $\beta$ be a countable ordinal $>\omega$ and $G$ a reduced $p$-group of Ulm type $\beta$. If $\beta$ is not a limit ordinal or $\beta$ is of type 1 then there exists a limit ordinal $\gamma<\beta$ such that $\gamma$ is of type 2 and $\gamma \omega>\beta$. In this case a theorem similar to Theorem 2.1 can be stated with the Ulm factors replaced by the $\omega \gamma$-factors, etc. If $\beta$ is a limit ordinal of type 2 , let $\left\{\alpha_{i}\right\}$ be an admissible sequence converging to $\beta$. Then a theorem similar to Theorem 2.8 can be stated for $\beta$-summable $p$-groups with the Ulm factors replaced by the $\left\{\alpha_{i}\right\}$-factors, etc.

LEMMA 3.4. Let $G$ be a reduced p-group of Ulm type $\beta$ such that $\beta$ is a countable limit ordinal of type $2($ or $\beta=\omega)$. Let $C$ be a $\beta$-summable subgroup of $G$. Then $C$ is $a$ dense subgroup of $G$ with respect to the $\beta$-topology on $G$.

Proof. Let $x \in G$ such that $O(x)=p^{k}$ and let $\tilde{\alpha}=\left\{\alpha_{i}\right\}_{i \in \omega}$ be a $(\beta-U)$-sequence. We will show that $(x+G\{\tilde{\alpha}\}) \cap C \neq \varnothing$. Let $\gamma$ be the least limit ordinal greater than or equal to $\alpha_{k}$. Then $\left(p^{\gamma} G\right)\left[p^{k}\right] \subset G\{\tilde{\alpha}\}$. (Note that $\gamma<\omega \beta$ since, otherwise, $\omega \beta=\alpha_{i}+\omega$.) Let $K$ be a $(\gamma+k)$-high subgroup of $C$, and let $M$ be a complementary summand of 
a maximal $p^{k}$-bounded summand of $p^{\gamma} G$. Then $G\left[p^{k}\right]=K\left[p^{k}\right] \oplus M\left[p^{k}\right]$ by Lemmas 1.1 and 1.10. Thus $x=y+z$ where $y \in K\left[p^{k}\right]$ and $z \in M\left[p^{k}\right]$. Thus $x+G\{\tilde{\alpha}\}=y+G\{\tilde{\alpha}\}$ and since $G\{\tilde{\alpha}\} \cap C \neq \varnothing$ we must have $(x+G\{\tilde{\alpha}\}) \cap C \neq \varnothing$.

LEMMA 3.5. Let $C$ be a p-group of length $\beta$, a countable limit ordinal of type 2 (or $\beta=\omega)$. Let $\bar{C}$ be the completion of $C$ with respect to the $\beta$-topology. Then $C$ is isotype in $\bar{C}$.

Proof. Suppose $p^{\alpha} C=C \cap p^{\alpha} \bar{C}$ for all $\alpha<\gamma$. If $\gamma$ is a limit ordinal then it is easily shown that $p^{\gamma} C=C \cap p^{\gamma} \bar{C}$. If $\gamma$ is not a limit ordinal then $\gamma=\alpha+1$ for some ordinal $\alpha$. Let $x \in C \cap p^{\gamma} \bar{C}$. Let $y \in p^{\alpha} \bar{C}$ such that $p y=x$. There exists a sequence $\left\{y_{i}\right\}_{i \in \omega}$ in $C$ converging to $y$. For sufficiently large $i, y_{i}-y \in p^{\alpha} \bar{C}$ and hence $y_{i} \in p^{\alpha} \bar{C} \cap C$ for these $i$. Therefore, $p y_{i}-x, p y_{i} \in p^{\gamma} C$ for large $i$; thus $x \in p^{\gamma} C$. The proof follows.

THEOREM 3.6. Let $G$ be a reduced p-group and let $\beta$ be a countable limit ordinal of type $2($ or $\beta=\omega)$. Let $C$ be a $\beta$-summable subgroup of $G$. Then $G / p^{\omega \beta} G$ is isomorphic to an isotype subgroup $V$ between $C$ and $\bar{C}$, the completion of $C$ with respect to the $\beta$-topology.

Proof. That $G / p^{\omega \beta} G$ is isomorphic to an isotype subgroup between $C$ and $\bar{C}$ follows from Lemmas 3.2 and 3.5 above and Proposition 5, p. 246 in [1].

THEOREM 3.7. Let $\beta$ be an ordinal of type 2 (or $\beta=\omega)$ and let $G$ be a reduced p-group of Ulm type $\geqq \beta$. Let $S$ be a subsocle of $G[p]$ dense with respect to the $\beta$-topology on $G$. Let $L$ be a subgroup of $G$ such that $L[p] \subset S$. Let $H$ be a maximal subgroup of $G$ containing $L$ and supported by $S$. Then $H$ is $p^{\omega \beta}$-pure $\left(^{1}\right)$ in $G$.

Proof. Let $\alpha<\omega \beta$. Since $S$ is dense in $G[p]$ we may write $G[p]=S \oplus P$ such that $P \subset p^{\alpha} G$. (If not, there exists $x \in G[p]-S$ such that the height of $x$ is $<\alpha$ and $x+\left(p^{\alpha} G\right)[p] \cap S=0$ contradicting the density of $S$.) By Theorem 2 of [8] we have that $H$ is $p^{\alpha+1}$-pure in $G$. Thus it follows easily that $H$ is $p^{\omega \beta}$-pure in $G$.

COROLlary 3.8. If the type of $G$ is $\leqq \beta$ then $H$ is isotype in $G$.

COROLlary 3.9. If $G$ is a group of type $\leqq \beta$ then $G$ and $H$ have the same (up to isomorphism) Ulm factors.

We will now proceed to give a general construction for groups of countable Ulm type. I.e., if $\tau$ is a countable ordinal, $\boldsymbol{W}$ is a cardinal, and $\left\{\boldsymbol{G}_{\alpha}\right\}_{\alpha \in \tau}$ is a sequence of $p$-groups without elements of infinite height satisfying

(i) $\sum_{0 \leqq \alpha<\tau}\left|G_{\alpha}\right| \leqq \boldsymbol{W} \leqq \prod_{0 \leqq \alpha<\min (\omega, \tau)}\left|G_{\alpha}\right|$,

(ii) $\sum_{\beta \leqq \alpha<\tau}\left|G_{\alpha}\right| \leqq\left|G_{\beta}\right|^{\aleph_{0}}$ for all $0 \leqq \beta<\tau$,

(iii) $r\left(B_{\alpha+1}\right) \leqq$ fin $r\left(G_{\alpha}\right)$ for all $1 \leqq \alpha+1<\tau$,

where $B_{\alpha+1}$ is a basic subgroup of $G_{\alpha+1}$, we will construct all reduced $p$-groups $G$ satisfying

(a) $|G|=\boldsymbol{N}$,

$\left.{ }^{1}\right)$ The terminology currently used is weakly $p^{\omega \beta}$-pure. 
(b) $\tau(G)=\tau$, and

(c) the Ulm sequence of $G$ is $\left\{G_{\alpha}\right\}_{\alpha \in \tau}$.

Let $\left\{G_{\alpha}\right\}_{\alpha \in \omega}$ be a sequence of $p$-groups with no elements of infinite height satisfying conditions (i) and (iii). For each $\alpha \in \omega$ let $B_{\alpha}$ be a basic subgroup of $G_{\alpha}$ such that $r\left(G_{\alpha} / B_{\alpha}\right) \geqq r\left(B_{\alpha+1}\right)$. Having chosen the $B_{\alpha}$ 's, let $H_{\alpha}$ be a pure subgroup of $G_{\alpha}$ containing $B_{\alpha}$ such that $r\left(G_{\alpha} / H_{\alpha}\right)=r\left(B_{\alpha+1}\right)$ and let $D_{\alpha+1}$ be a divisible hull of $B_{\alpha+1}$. Let $K_{\alpha}$ be a subdirect sum of $G_{\alpha}$ and $B_{\alpha+1}$ with high subgroup $H_{\alpha}$ and elements of infinite height $B_{\alpha+1}$. Let $C=\left(\sum_{\alpha \in \omega} K_{\alpha}\right) / R$ where $R$ is the relation that identifies $p^{\omega}\left(K_{\alpha}\right)$ with the basic subgroup $B_{\alpha+1}$ in $K_{\alpha+1}$. Let $\bar{C}$ be the completion of $C$ with respect to the $\omega$-topology. Let $S$ be a subsocle of $\bar{C}[p]$ containing $C[p]$, with $|S|=\boldsymbol{\aleph}$, and let $G$ be an isotype subgroup of $\bar{C}$ supporting $S$. Then $G$ is a reduced $p$-group satisfying conditions (a)-(c). Using the results of $\$ 1$ and 2 we note that all reduced $p$-groups of type $\leqq \omega$ can be constructed in this manner.

Next let $\beta$ be a countable ordinal $>\omega$ and suppose that all $p$-groups of type $<\beta$ have been constructed, and, moreover, if $\gamma<\beta$ and $\left\{G_{\alpha}\right\}_{\alpha \in \gamma}$ is a sequence of $p$-groups without elements of infinite height satisfying conditions (i)-(iii), then a group $G$ has been constructed satisfying conditions (a)-(c) (one may take $|G|<\boldsymbol{W}$ where appropriate).

Let $\left\{\boldsymbol{G}_{\alpha}\right\}_{\alpha \in \beta}$ be a sequence of $p$-groups with no elements of infinite height satisfying conditions (i)-(iii).

If $\beta$ is not a limit ordinal or if $\beta$ is an ordinal of type 1 , let $\rho$ be a limit ordinal of type 2 (or $\rho=\omega$ ) such that $\rho<\beta$ and $\rho \omega>\beta$ (the least limit ordinal $\rho$ such that $\rho \omega \geqq \beta$ will do). Let $n$ be the least positive integer such that $\rho n \geqq \beta$. Let $C_{i}$ for $i=0, \ldots, n-2$ be a $\rho$-summable reduced $p$-group such that the Ulm sequence of $C_{i}$ is $\left\{G_{\alpha}\right\}_{\rho i \leqq \alpha<\rho(i+1)}$ and $\left|C_{i}\right| \leqq \mathcal{N}$, and let $G_{n-1}$ be a reduced $p$-group such that the Ulm sequence of $G_{n-1}$ is $\left\{G_{\alpha}\right\}_{\rho(n-1) \leqq \alpha<\beta}$ and $\left|G_{n-1}\right| \leqq \aleph$. Next let $\bar{C}_{i}$ be the completion of $C_{i}$ with respect to the $\rho$-topology and let $G_{i}$ be an isotype subgroup of $\bar{C}_{i}$ containing $C_{i}$ such that $\left|G_{i}\right| \leqq \mathcal{K}$ and $r\left(G_{i} / C_{i}\right) \geqq r\left(B_{i+1}\right), B_{i+1}$ a basic subgroup of $C_{i+1}$ (or $G_{n-1}$ if $i=n-2$ ). In the choice of the $G_{i}$ 's we must choose at least one with cardinality $\boldsymbol{\aleph}$. Let $H_{i}$ be an isotype subgroup of $G_{i}$ containing $C_{i}$ such that $r\left(G_{i} / H_{i}\right)=r\left(B_{i+1}\right), i=0, \ldots, n-2$. Let $K_{i}$ be the subdirect sum of $G_{i}$ and $B_{i+1}$ with $\rho$-high subgroup $H_{i}$ and $p^{\omega \rho} K_{i} \cong B_{i+1}$ for $i=0, \ldots, n-2$, and let $K_{n-1}=G_{n-1}$. Let $G=\left(\sum_{i=0}^{n-1} K_{i}\right) / R$ where $R$ is the relation that identifies $p^{\omega \rho} K_{i}$ with $B_{i+1}$ the basic subgroup of $G_{i+1}$. Then $G$ satisfies conditions (a)-(c).

If $\beta$ is an ordinal of type 2 , let $\left\{\alpha_{i}\right\}$ be an admissible sequence converging to $\beta$. Let $\left\{C_{i}\right\}_{i \in \omega}$ be a sequence of reduced $p$-groups such that $C_{i}$ is $\left(\alpha_{i+1}-\alpha_{i}\right)$-summable, the Ulm sequence of $C_{i}$ is $\left\{G_{\alpha}\right\}_{\alpha_{i} \leqq \alpha<\alpha_{i+i}}$ and $\left|C_{i}\right| \leqq \mathcal{N}$. For each $i \in \omega$ let $B_{i}$ be a basic subgroup of $C_{i}$, let $\bar{C}_{i}$ be the completion of $C_{i}$ with respect to the $\left(\alpha_{i+1}-\alpha_{i}\right)$ topology, and let $G_{i}$ be an isotype subgroup of $\bar{C}_{i}$ containing $C_{i}$ such that $r\left(G_{i} / C_{i}\right)$ $\geqq r\left(B_{i+1}\right)$ and $\left|G_{i}\right| \leqq \aleph$. For each $i \in \omega$ let $H_{i}$ be an isotype subgroup of $G_{i}$ containing $C_{i}$ such that $r\left(G_{i} / H_{i}\right)=r\left(B_{i+1}\right)$ and let $D_{i+1}$ be a divisible hull of $B_{i+1}$. Let $K_{i}$ be a subdirect sum of $G_{i}$ and $B_{i+1}$ with $\left(\alpha_{i+1}-\alpha_{i}\right)$-high subgroup $H_{i}$ and 
$p^{\omega\left(\alpha_{i+1}-\alpha_{i}\right)} G_{i} \cong B_{i+1}$. Let $C=\left(\sum G_{i}\right) / R$ where $R$ is the relation that identifies the $p^{\omega\left(\alpha_{i+1}-\alpha_{i}\right)} G_{i}$ 's with $B_{i+1}$, the basic subgroup of $G_{i+1}$. Let $\bar{C}$ be the completion of $C$ with respect to the $\beta$-topology, and let $G$ be an isotype subgroup of $\bar{G}$ containing $C$ such that $|G|=\aleph$. Then $G$ satisfies conditions (a)-(c) and we see from $\S 3$ that every group of length $\beta$ is obtainable in this manner.

4. Examples. The following facts will be needed to justify some of the statements in the construction of the examples.

Lemma 4.1. Let $V$ be a vector space over a field $F$ with infinite dimension $r(V)$. Let $V=M \oplus N$ such that $M \neq 0$ and $r(N)=m$ an infinite cardinal number. Then there exists at least $2^{m}$ (distinct) complementary summands of $M$ in $V$.

Proof. Let $N=\sum_{\alpha \in \beta}\left\langle x_{\alpha}\right\rangle$ with $|\beta|=m$. Let $0 \neq x \in M$. Let

$$
\mathscr{S}=\left\{\left\{z_{\alpha} \mid \alpha \in \beta\right\} \mid z_{\alpha} \in\left\{x_{\alpha}, x_{\alpha}+x\right\} \text { for each } \alpha \in \beta\right\} .
$$

Then $|\mathscr{S}|=2^{m}$; if $L, L^{\prime} \in \mathscr{S}$ such that $L \neq L^{\prime}$ then $\langle L\rangle \neq\left\langle L^{\prime}\right\rangle$; and for each $L \in \mathscr{S}$, $V=M \oplus \sum_{x \in L}\langle x\rangle$.

COROLLARY 4.2. If $G$ is an unbounded reduced p-group with countable basic subgroup such that $|G[p]|=m$, then there exists $2^{m}$ dense subsocles $Q$ of $G[p]$ such that $G[p] / Q$ has rank one. In fact there exists $2^{m}$ such subsocles containing the socle of the same basic subgroup.

Proposition 4.3. Let $\bar{B}$ be a closed p-group with unbounded countable basic subgroup $B$. Then there exists a set $\mathscr{G}$ of $2^{c}$ nonisomorphic pure subgroups between $B$ and $\bar{B}$ such that

(1) if $G \in \mathscr{G}$ then $\bar{B} / G \cong Z\left(p^{\infty}\right)$;

(2) if $G, H \in \mathscr{G}, G \neq H$, then $G[p] \neq H[p]$;

(3) if $G, H \in \mathscr{G}, G \neq H$, there does not exist an automorphism $\varphi$ of $\bar{B}$ such that $\varphi(G[p])=H[p]$.

Proof. Since there are at most $c$ automorphisms of $\bar{B}$ (see [5]) the theorem follows from Corollary 4.2.

EXAMPLE 1. Let $G$ and $H$ be reduced $p$-groups with high subgroups $K$ and $L$, respectively, such that $G / p^{\omega} G \cong H / p^{\omega} H$ under an isomorphism $\varphi, p^{\omega} G \cong p^{\omega} H$, and if $\sigma: G \rightarrow G / p^{\omega} G$ and $\rho: H \rightarrow H / p^{\omega} H$ are natural then $\varphi(\sigma(H))=\rho(L)$. If $\rho^{\omega} G$ is a direct sum of cyclic groups all of the same order, it follows by Theorem 1 that $G$ and $H$ are isomorphic. The following example will show that we cannot remove the restriction that $p^{\omega} G$ be a direct sum of cyclic groups all of the same order and have $G$ and $H$ necessarily isomorphic. In fact, if $p^{\omega} G \cong p^{\omega} H \cong\left\langle x_{1}\right\rangle \oplus\left\langle x_{2}\right\rangle$ such that $O\left(x_{i}\right)=p^{i}$ for $i=1,2$ then there exists such nonisomorphic groups $G$ and $H$.

Let $B=\sum_{i=1}^{\infty}\left\langle b_{i}\right\rangle$ such that $O\left(b_{i}\right)=p^{i}$. Let $\bar{B}$ be the torsion completion of $B$. Let $Q$ and $R$ be subsocles of $\bar{B}$ containing $B[p]$ such that

(1) $\bar{B}[p] / Q \cong \bar{B}[p] / R \cong Z(p)$ and

(2) there exists no automorphism $\varphi$ of $\bar{B}$ such that $\varphi(Q)=R$. 
Let $L$ be a pure subgroup of $\bar{B}$ such that $L[p]=R \cap Q$. Let $K_{Q}$ and $K_{R}$ be pure subgroups of $\bar{B}$ containing $L$ such that $K_{Q}[p]=Q$ and $K_{R}[p]=R$. Let $M=\left\langle x_{1}\right\rangle$ $\oplus\left\langle x_{2}\right\rangle$ such that $O\left(x_{i}\right)=p^{i}, i=1,2$. Let $D_{i}$ be a divisible hull of $\left\langle x_{i}\right\rangle, i=1,2$, and let $D=D_{1} \oplus D_{2}$. Now $K_{Q}[p]=L[p] \oplus\langle z\rangle, K_{R}[p]=L[p] \oplus\langle w\rangle$, and $\bar{B}[p]=L[p]$ $\oplus\langle z\rangle \oplus\langle w\rangle$. Let $d_{i} \in D_{i}, i=1,2$, such that $p d_{i}=x_{i}$. Define isomorphisms $\varphi_{1}$ : $\bar{B} / L \rightarrow D / M$ such that $\varphi_{1}(z+L)=d_{1}+M, \varphi_{1}(w+L)=d_{2}+M$, and $\varphi_{1}\left(K_{Q} / L\right)=D_{1}$ and $\varphi_{2}: \bar{B} / L \rightarrow D / M$ such that $\varphi_{2}(z+L)=d_{2}+M, \varphi_{2}(\omega+L)=d_{1}+M$, and $\varphi_{2}\left(K_{R} / L\right)=D_{1}$.

Let $\alpha: \bar{B} \rightarrow \bar{B} / L$ and $\beta: D \rightarrow D / M$ be natural. Define subgroups $G$ and $H$ of $\bar{B} \oplus D$ as $G=\left\{(b, d) \mid b \in \bar{B}, d \in D\right.$ and $\left.\varphi_{1} \alpha(b)=\beta(d)\right\}$ and $H=\{(b, d) \mid b \in \bar{B}, d \in D$ and $\left.\varphi_{2} \alpha(b)=\beta(d)\right\}$. Thus $p^{\omega} G=\{0\} \oplus M \cong p^{\omega} H \cong M, G / p^{\omega} G \cong H / p^{\omega} H \cong \bar{B}, L \oplus\{0\}$ is a high subgroup of $G$ and $H$, and any isomorphism between the embeddings of high subgroups of $G$ and $H$ in $G / p^{\omega} G$ and $H / p^{\omega} H$, respectively, extends to an isomorphism between $G / p^{\omega} G$ and $H / p^{\omega} H$ (see [11]).

Suppose that $\varphi$ is an isomorphism from $G$ onto $H$. Note that

$$
\hat{K}_{Q}=\left\{(k, d) \in G \mid k \in K_{Q} \text { and } d \in D_{1}\right\}
$$

is $\left\langle x_{2}\right\rangle$-high in $G ; \sigma\left(\hat{K}_{Q}\right)=K_{Q}$ where $\sigma: G \rightarrow G / p^{\omega} G$ is natural;

$$
\hat{K}_{R}=\left\{(k, d) \in H \mid k \in K_{R} \text { and } d \in D_{1}\right\}
$$

is $\left\langle x_{2}\right\rangle$-high in $H$; and $\gamma\left(\hat{K}_{R}\right)=K_{R}$ where $\gamma: H \rightarrow H / p^{\omega} H$ is natural. Now $\varphi\left(\hat{K}_{Q}\right)$ is $\left\langle x_{2}\right\rangle$-high in $L$ and $\gamma\left(\varphi\left(R_{Q}\right)\right)[p]=\gamma\left(R_{R}\right)[p]$ (by Lemma 4 in [8]). Hence $\varphi$ induces an automorphism of $\bar{B}$ taking $K_{Q}[p]$ onto $K_{R}[p]$ which is a contradiction. Thus $G$ and $L$ are not isomorphic.

EXAmple 2. This example shows that the connection between the Ulm factors described in conditions (b) and (c) of Definition 2.2 must be given.

Let $G$ be a reduced $p$-group such that $G / p^{\omega} G \cong p^{\omega} G \cong \bar{B}$ where $B$ is a countable unbounded direct sum of cyclic groups. Let $\left\{G_{i}\right\}_{i \in \omega}$ be a high chain in $G$. Let $G_{\omega}=\bigcup_{i=0}^{\infty} G_{i}$. Let $x \in p^{\omega} G[p]-p^{\omega} G_{\omega}[p]$. Let $H$ be an isotype subgroup of $G$ containing $G_{\omega}$ such that $H[p]=G_{\omega}[p] \oplus\langle x\rangle$. Now by [5] there exists $2^{c}$ nonisomorphic pure subgroups $K$ of $H$ such that $K[p]=G_{\omega}[p]$ and $p^{\omega} G_{\omega} \subset K$. Note that $K / p^{\omega} K \cong G_{\omega} / p^{\omega} G_{\omega} \cong H / p^{\omega} H$. Thus some $K$ is not isomorphic to $H$. But if $\left\{K_{i}\right\}_{i \in \omega}$ is a high chain in $K$, then $K_{i}$ is $(\omega+i)$-high in $H$. If $\sigma: H \rightarrow H / p^{\omega} H$ and $\sigma^{\prime}: K \rightarrow K / p^{\omega} K$ are natural, then $\varphi: K / p^{\omega} K \rightarrow H / p^{\omega} H: k+p^{\omega} K \rightarrow k+p^{\omega} H$ is an isomorphism such that $\varphi\left(\sigma^{\prime}\left(K_{i}\right)\right)=\sigma\left(K_{i}\right)$. Also $p^{\omega} K$ and $p^{\omega} H$ are isomorphic. But $K$ and $H$ are not isomorphic. Thus the connection must be prescribed.

\section{REFERENCES}

1. N. Bourbaki, General topology. Part I, Hermann, Paris and Addison-Wesley, Reading, Mass., 1966. MR 34 \#5044a.

2. B. Charles, Sous-groupes fonctoriels et topologies, Studies on Abelian Groups, Sympos. (Montpellier, 1967), Springer-Verlag, Berlin and New York, 1968, pp. 75-92. MR 39 \#1547.

3. D. O. Cutler and R. W. Stringall, A topology for primary abelian groups, Studies on Abelian Groups, Sympos. (Montpellier, 1967), Springer-Verlag, Berlin and New York, 1968. MR 39 \#1546. 
4. L. Fuchs, Abelian groups, Publ. House Acad. Sci., Budapest, 1958. MR 21 \#5672.

5. P. Hill and C. Megibben, On primary groups with countable basic subgroups, Trans. Amer. Math. Soc. 124 (1966), 49-59. MR 33 \#7409.

6. J. M. Irwin, High subgroups of Abelian groups, Pacific J. Math. 11 (1961), 1375-1384. MR 25 \#119b.

7. J. M. Irwin and E. A. Walker, On N-high subgroups of Abelian groups, Pacific J. Math. 11 (1961), 1363-1374. MR 25 \#119a.

8. - On isotype subgroups of Abelian groups, Bull. Soc. Math. France 89 (1961), 451-460. MR 26 \#5054.

9. E. Kamke, Theory of sets, Dover, New York, 1950. MR 11, 335.

10. I. Kaplansky, Infinite abelian groups, Univ. of Michigan Press, Ann Arbor, Mich., 1954. MR 16, 444.

11. H. Leptin, Zur Theorie der überabzählbaren abelschen p-Gruppen, Abh. Math. Sem. Univ. Hamburg 24 (1960), 79-90. MR 23 \#A207.

UNiVersity OF CALIFORNia,

Davis, California 95616 